rev.relac.int.estrateg.segur.10(2):155-178,2015

\title{
¿ES POSIBLE LA CONVERGENCIA EN LA DIVERSIDAD?: CHILE ENTRE LA ALIANZA DEL PACÍFICO Y EL MERCOSUR*
}

\author{
Gilberto Aranda Bustamante** \\ Jorge Riquelme Rivera***
}

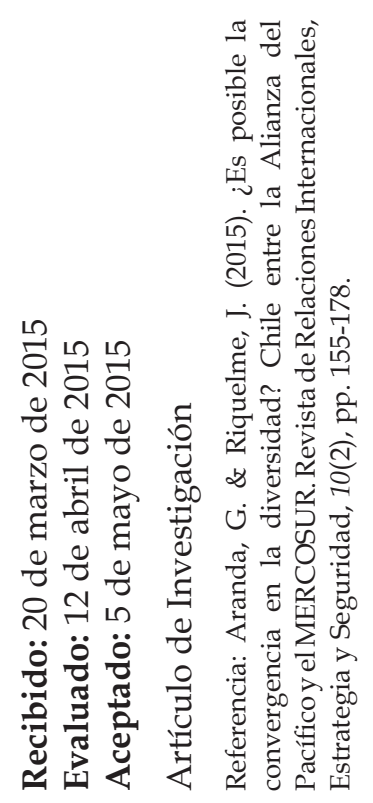

\section{RESUMEN}

El artículo analiza la iniciativa de Chile de impulsar la convergencia entre la Alianza del Pacífico y el MERCOSUR, planteada por el actual gobierno de Michelle Bachelet, que se enmarca en la prioridad regional declarada por la política exterior de dicho país. Para tal efecto, a partir de una revisión bibliográfica y de archivos de prensa sobre la materia, se examinan las tendencias que ha seguido el proceso de

Este trabajo forma parte del Proyecto de Iniciación FONDECYT (Chile) №11130638 "La influencia de las corrientes partidarias y los liderazgos nacionales en la arquitectura institucional regional".

** Doctor en estudios latinoamericanos, Universidad de Chile. Académico del Instituto de Estudios Internacionales de la Universidad de Chile e Investigador del Instituto de Estudios Internacionales de la Universidad Arturo Prat. Correo electrónico: garanda@uchile.cl.

*** Candidato a Doctorado en Relaciones Internacionales, Universidad Nacional de la Plata (UNLP). Graduado del Centro de Estudios Hemisféricos de Defensa (Universidad de la Defensa Nacional, Estados Unidos de América). Docente de la Escuela de Ciencia Política de la Universidad Diego Portales, Chile. Correo electrónico: jlriquel@uc.cl. 
integración latinoamericano y las opciones que ha seguido el país andino al respecto, así como comparar ambos bloques. El trabajo concluye que, más que reflexionar respecto de la posibilidad de tal convergencia, cabe considerar este proceso como una necesidad, en virtud de favorecer los intereses de la región en materia de inserción internacional.

Palabras clave: Alianza del Pacífico, Convergencia en la Diversidad, MERCOSUR, Política Exterior de Chile.

\title{
IS IT POSSIBLE CONVERGENCE WITHIN DIVERSITY?: CHILE BETWEEN THE ALLIANCE OF THE PACIFIC AND MERCOSUR
}

\begin{abstract}
The article analyses Chile's initiative to encourage convergence between Alliance of the Pacific and MERCOSUR, proposed by Michelle Bachelet's government within the framework of the regional priority declared by Chile's foreign policy. To this end, reviewing literature and press, this work examines the trends occurred in the Latin American integration process and the options followed by Chile in this respect, while comparing both blocks. The paper concludes that, in addition to reflecting on the possibility of convergence, it is essential to consider this process as a necessity, in order to favor the interests of the region on international insertion.
\end{abstract}

Keywords: Alliance of the Pacific, Convergence within Diversity, MERCOSUR, Chilean Foreign Policy.

\section{É POSSÍVEL A CONVERGÊNCIA NA DIVERSIDADE?: O CHILE ENTRE A ALIANÇA DO PACÍFICO E O MERCOSUL}

\section{RESUMO}

O artigo analisa a iniciativa do Chile de impulsar a convergência entre a Aliança do Pacífico e o MERCOSUL, formulada pelo atual Governo de Michelle Bachelet, que se insere na prioridade regional declarada pela política exterior do país. Para tanto, a partir de uma revisão bibliográfica e dos arquivos de imprensa sobre a matéria, examinam-se as tendências que o país andino tem seguido a respeito, assim como se comparam os dois blocos. O trabalho conclui que, mais do que refletir a respeito da possibilidade de tal convergência, cabe considerar este processo como uma necessidade em virtude de favorecer os interesses da região em questão na inserção internacional.

Palavras-chave: Aliança do Pacífico, Convergência na Diversidade, MERCOSUL, Política Exterior do Chile. 


\section{INTRODUCCIÓN}

A lo largo de su historia republicana, América Latina ha visto desarrollar diversos procesos de integración, que tienen su antecedente remoto en el Congreso Anfictiónico de Panamá de 1826, impulsado por Simón Bolívar ante las tendencias hegemónicas que exhibía Estados Unidos con la Doctrina Monroe de 1823. En la actualidad, coexisten variados procesos en marcha, con distintos objetivos y resultados, como es el caso del Mercado Común del Sur (MERCOSUR), la Comunidad Andina (CAN) y la Unión de Naciones Suramericanas (UNASUR).

No deben dejar de mencionarse ciertos procesos que ya a primera vista aparecen antagónicos en sus principios, en cuanto responden a diversos intereses políticos y económicos (Aranda \& Salinas, 2015), como es el caso de la aperturista Alianza del Pacífico y la proteccionista Alianza Bolivariana de los Pueblos de Nuestra América (ALBA). Tales intentos cuentan entre sus antecedentes pioneros los avances que implicó la Asociación Latinoamericana de Libre Comercio (ALALC) y la posterior Asociación Latinoamericana de Integración (ALADI).

Considerando este confuso panorama, el presente trabajo pretende, a través del análisis de la bibliografía disponible, fuentes oficiales y archivos de prensa sobre la materia, analizar las tendencias que ha seguido la política exterior de Chile en materia de integración regional, poniendo un especial énfasis en la iniciativa del segundo gobierno de Michelle Bachelet, respecto al establecimiento de puentes entre el MERCOSUR y la Alianza del Pacífico, buscando en último término, la denominada convergencia en la diversidad, planteada en los albores de la actual administración por el Ministro de Relaciones Exteriores de Chile, Heraldo Muñoz (2014).

Para los efectos de este trabajo, se entenderá la política exterior como la totalidad de las acciones y no acciones, reacciones y proacciones, interacciones, y contactos tomados por un Estado soberano hacia otra u otras entidades soberanas, o conducidas entre un Estado soberano y otro u otros Estados (Nanjira, 2010). Es decir, corresponde a aquella política pública que guía la conducta de un Estado en el ámbito internacional, con el objeto de apoyar su posicionamiento en el mismo, considerando sus particulares intereses y objetivos nacionales, así como los medios y estrategias para alcanzarlos. En suma, según señala Alberto Van Klaveren, "por medio de la política exterior, los Estados se relacionan con el sistema internacional y se adaptan a éste" (2014, p.96).

Asimismo, se utiliza el concepto del regionalismo. Para entenderlo, primero hay que hacerse cargo de la idea de región, que apunta a una experiencia histórica común, un sentimiento compartido frente a los problemas comunes internos o amenazas externas, que van generando una percepción de identidad y conciencia regional (Hurrell, 1995). Si los vínculos se estrechan, se puede observar una frontera imaginaria al interior de la cual las interacciones son más intensas que aquellas con en el mundo circundante. Posteriormente, se institucionaliza el proceso en el que la región adopta un sentido de configuración normativa, confiriéndose reglas del juego precisas al interior de la región, surgiendo el denominado regionalismo (Morandé, 1999). A 
este respecto, Bernal-Meza sugiere que son las diferencias de estas identidades las que fungen como unidades de análisis para los especialistas (2011), en una ecuación que coloca los énfasis en la proximidad e intensidad de una relación construida socialmente, que desemboca en un tipo de regionidad (regionness), si seguimos a Hettne \& Söderbaum (2002).

Desde esta perspectiva, se puede referir en América Latina a un primer y a un segundo regionalismo. El primero de ellos posee las características propias de la integración regional de los sesenta y setenta, es decir, con un componente de desarrollo hacia adentro, signado por el proteccionismo, con el Mercado Común Centroamericano (MCCA) de 1960, el Pacto Andino en 1969 y la Comunidad del Caribe (CARICOM) de 1973, como preclaros ejemplos. En tanto que el segundo regionalismo, también conocido como neo-regionalismo, que despunta principalmente en la década de los noventa, es un proceso exógeno que incentiva la integración de la región en el mundo (Bouzas, 1997), empujando el nucleamiento regional en torno a la estrategia del regionalismo abierto. Tal concepto se distingue del viejo regionalismo, por una vocación manifiesta a la negociación comercial con otros mercados extra-regionales en una malla de acuerdos que se superponen, siempre abiertos a la potencial adhesión de nuevos participantes (Bernal-Meza, 2013).

El nuevo siglo trajo una divergencia gradual, aunque creciente de la región, ante tales tendencias. Una parte relevante de los gobiernos comienzan a sostener políticas contrarias a la dinámica económica de los años anteriores, donde los procesos de integración, como el MERCOSUR, empiezan a incorporar en su agenda variables sociales, como la educación, la cultura y medidas contra la desigualdad. En este marco, nace el año 2005 la UNASUR, que proyecta una integración de carácter político y social más que económico, desalojando a los temas comerciales de la cabecera de la agenda. Algunos analistas advierten que a partir de ALBA y la UNASUR se habría dado inicio a la era de los regionalismos "pos" (Perrotta, 2013), posliberal con primacía de la agenda política (Sanahuja, 2008), posneoliberal (Bizzozero, 2011) o simplemente poshegemónico, que sitúa al diálogo político de alto nivel (presidencial) en la plataforma instrumental predilecta para la generación de entendimientos compartidos para materializar lo que a arquitectura institucional se refiere. Desde dicha dinámica los temas comerciales son subordinados en favor de la centralidad de la dimensión política.

Junto con lo anterior, aparecen otras lecturas -más cercanas al neo funcionalismo- sobre el tipo de integración regional a seguir en un cuadro claramente divergente (Malamud, 2009), signado por la fragmentación subregional y la sobreoferta de propuestas integradoras (Rojas, 2013, p.166). Desde otro registro, Briceño constata esta divisoria de aguas regional en torno a tres ejes: aperturista, revisionista y anti-sistémico (Briceño, 2013), representados en los bloques de la Alianza del Pacífico, MERCOSUR y ALBA.

Ciertamente, la construcción de la integración ha significado igualmente conflictos por el liderazgo regional, según señala Nolte $(2006 ;$ 2007), como lo evidencia la competencia entre el papel de Estados Unidos en la región, con iniciativas tales como la Alianza para el Progreso 
en los sesenta o el Área de Libre Comercio de las Américas hacia el cierre de la centuria pasada, y el prominente papel de Brasil en Sudamérica, plasmado en el proyecto de la UNASUR. Sin embargo, pensamos que los actuales regionalismos, reflejados en los referidos bloques, sobretodo suponen divergencias en la inserción internacional y la estrategia de desarrollo de los países (Actis, 2014; Busso, 2014).

Bajo las premisas de la conceptualización anterior, el trabajo se enfocará en las posibilidades de la señalada propuesta de la política exterior del nuevo gobierno chileno de Michelle Bachelet, inaugurado el 11 de marzo de 2014. Para tal efecto, el artículo primero se refiere a las tendencias que ha seguido Chile en materia de integración regional, luego se enfoca en las características y desarrollo de la Alianza del Pacífico y el MERCOSUR, y finalmente reflexiona sobre la señalada iniciativa de la política exterior de Chile para impulsar la convergencia entre ambos bloques.

\section{CHILE Y LA INTEGRACIÓN REGIONAL}

El país ha participado, con diversos grados de profundidad, en varias instancias regionales, como es el caso del MERCOSUR, la CAN y la UNASUR (ocupando la Secretaría Pro Témpore de esta instancia durante el año 2008 y parte del 2009). También cabe destacar la contribución de Chile en el proceso de convergencia del Grupo de Río y la Cumbre de América Latina y el Caribe (CALC), que decantó en la Comunidad de Estados Latinoamericanos y Caribeños (CELAC), presidida por este país entre diciembre de 2011 y enero de 2013.

Haciendo un poco de historia, es necesario señalar que los impulsos de apoyo a la integración regional fueron particularmente visibles durante la década de los sesenta, cuando el país fue un activo y voluntarista promotor del regionalismo, lo que se plasmó en el protagonismo chileno en la constitución del Pacto Andino. A ello contribuyó el fuerte influjo de las ideas de la Comisión Económica para América Latina (CEPAL), que concebían en ese entonces a la integración como un mecanismo defensivo frente al capitalismo internacional que, al tiempo que favorecía la ampliación de los mercados nacionales, proyectaba un esquema de industrialización por sustitución de importaciones a la escala regional.

Estas tendencias cambiaron desde 1973, cuando el golpe militar inauguró un régimen contrario a toda tendencia latinoamericanista. La integración regional pasó a percibirse como un asunto propio de la izquierda, bajo el prisma de la doctrina de seguridad nacional. Ello se expresó, por ejemplo, en el retiro del país del Pacto Andino. La integración regional también parecía contrarrevolucionaria en relación con el intenso proceso de liberalización económica que se puso en marcha en el país en ese período.

Este enfoque nuevamente cambió con la vuelta de la democracia en Chile, que trajo consigo un urgente proceso de reinserción internacional y regional. Aunque esta vez la mirada sobre la integración regional fue más pragmática y menos voluntarista que en los años sesenta, en 
virtud del principio del regionalismo abierto, que ha constituido la impronta fundamental de la diplomacia del país desde la década de los noventa, así como por la trascendencia del comercio exterior para el modelo de desarrollo chileno (Wilhelmy \& Durán, 2003). Entre los intereses prioritarios de la política exterior está el contribuir al fortalecimiento de la integración regional. En esta línea, se sostiene que:

La integración de Chile a América Latina se ha constituido en uno de los elementos más importantes de nuestra política exterior, lo cual se refleja en el significativo número de proyectos que, en diferentes áreas, se orientan a acercarnos a los países de la región bajo un marco de un mutuo respeto y entendimiento (Ministerio de Relaciones Exteriores, 2010, p.21).

Si bien existen variados y a veces contradictorios procesos de integración en la región, las tendencias que ha seguido el país en la materia han llevado a Carlos Portales a sostener que "la política latinoamericana de Chile ha contribuido a limar asperezas entre los países de los mecanismos en que participa y a buscar consenso para avanzar, hasta donde sea posible alcanzarlos" (2011, p.178). Asimismo, el acercamiento a la región, en el marco de la plural inserción internacional del país, responde a que las exportaciones de mayor valor agregado del país se dirigen justamente a países latinoamericanos.

Esta posición moderada de Chile ante la integración regional responde a su interés por incrementar su estatura estratégica, autonomía relativa e influencia en el multilateralismo internacional. Es decir, como un país con pocos recursos de poder en su sentido más tradicional y con un pequeño tamaño relativo, su participación en instancias de integración regional se aprecia como una manera pragmática de incrementar su peso y capacidad de incidencia en las decisiones mundiales. Desde una perspectiva comercial, la integración:

obedece a una estrategia de inserción política y económica de carácter global, pues responde al interés chileno por fomentar un entorno regional estable para el comercio que permita, entre otros aspectos, plantear a Chile como un puente estratégico entre el Océano Atlántico y el Pacífico. (Aranda \& Riquelme, 2011)

El interés chileno en constituirse como país puente entre la región y el Asia Pacífico, implica igualmente apoyar el fortalecimiento de la interconectividad y la infraestructura de transportes y telecomunicaciones en la región. Lo anterior supone que Chile participe activamente en la iniciativa para la Integración de la Infraestructura Regional Sudamericana (IIRSA), creada en agosto del año 2000, con el fuerte impulso de Brasil, en el marco de la primera Cumbre Sudamericana.

Al menos en el plano discursivo, las tendencias hacia la región mantuvieron una continuidad durante la presidencia de derecha de Sebastián Piñera, apoyado por la denominada Alianza por Chile. En su programa de Gobierno, se señalaba que "América Latina debe constituir 
una prioridad en nuestra política exterior, en orden a generar acuerdos estratégicos y una mayor integración, sin que ello implique la renuncia a nuestro exitoso modelo de desarrollo económico y social" (Piñera, 2010). No obstante, Piñera fue enfático en criticar la proliferación de iniciativas de integración regional, a su juicio, muchas veces superpuestas, con un bajo nivel de coordinación y que tienden a la duplicación de esfuerzos. En esta línea, en la víspera de la Cumbre Iberoamericana, celebrada en Paraguay en octubre de 2011, Piñera cuestionó la capacidad de los foros regionales para alcanzar efectivamente la integración, señalando que "nuestro continente todavía tiene muchas instituciones, muchas organizaciones, pero poca integración real. Como Presidente, a uno lo invitan a las cumbres, y yo digo que no parecen cumbres, ya parecen cordilleras" (La Tercera, 2011, 28 de octubre).

Pese a lo antes señalado sobre las tendencias del Gobierno de Piñera en materia de integración regional, lo cierto es que su gobierno fue fuertemente criticado por priorizar tratados y acuerdos comerciales con países extra regionales, sin estrechar lazos más estratégicos y políticos con los países latinoamericanos. En todo caso, se trata de una antigua crítica general que se le ha hecho a Chile desde el retorno al régimen civil, pese a que fue particularmente notable durante la administración Piñera.

Un aspecto que cabe resaltar en la misma línea, es el distanciamiento que se evidenció en las relaciones del gobierno de Piñera con el entorno vecinal y paravecinal. Ello fue particularmente complejo en las relaciones con Bolivia, que llevó su pretensión marítima a la Corte de La Haya. Lo mismo respecto de Perú, con el cual las relaciones fueron manejadas bajo el muy criticado concepto de las "cuerdas separadas", es decir, procurando que el complejo tema de la demanda peruana ante la Corte de La Haya no afectara la dinámica de las relaciones comerciales.

Por su parte Ecuador, considerado un aliado histórico, no apoyó a Chile durante el juicio. Respecto de Brasil, las relaciones fueron distantes, pese a los empeños de acercamiento del gobierno chileno. Lo mismo sucedió con la Argentina de Cristina Fernández, el país con el que históricamente Chile más ha avanzado en cooperación e integración.

Esta compleja situación regional y vecinal estuvo al centro de la crítica que expresó Michelle Bachelet, en materia de política exterior durante la campaña presidencial. En la sección dedicada a las Relaciones Exteriores del programa de gobierno 2014-2018, se señala entre otros elementos que:

Debemos fortalecer nuestra participación en los distintos mecanismos de integración actualmente existentes en América Latina y, en especial en América del Sur...Chile ha perdido presencia en la región, sus relaciones vecinales son problemáticas, se ha impuesto una visión mercantil de nuestros vínculos latinoamericanos y se han ideologizado las opciones de inserción externa. (Bachelet, 2013, p.154) 
Ya en los primeros días de su nueva administración, en un evento celebrado en la CEPAL, la ahora Presidenta Michelle Bachelet planteó que "no hago más que constatar un hecho si digo que Chile, en los últimos años, ha perdido presencia regional y que en sus vínculos se ha priorizado excesivamente una visión economicista" (La Tercera, 2014, 12 de marzo).

De todos modos, cabe anotar que una debilidad que aún se aprecia en esta línea es la carencia de una institucionalidad fuerte para tratar el tema de la integración en el Ministerio de Relaciones Exteriores chileno. A diferencia de otras Cancillerías, como en Argentina, Brasil o Ecuador, que cuentan con direcciones o reparticiones de alto perfil encargadas del seguimiento de esta temática, en Chile es sólo durante el actual gobierno de Bachelet cuando se crea la Dirección de Integración Regional Multilateral, que de todas maneras cuenta con una baja dotación de personal. Anteriormente, el tema estaba disperso en varias unidades del Ministerio, sin un seguimiento especializado y coherente con la declarada prioridad regional de la política exterior. Este es un tema todavía pendiente, que debiera asumirse en lo que resta de la actual administración, especialmente ante el desarrollo, variedad y complejidad que expresan los diversos mecanismos de integración existentes, los que requieren de un seguimiento especializado en los aspectos políticos y sectoriales.

\section{LA ALIANZA DEL PACÍFICO}

La vinculación con el área del Océano Pacífico es una de las continuidades de más largo aliento en la historia republicana chilena. Desde Diego Portales al Presidente Balmaceda en el siglo XIX, diversos líderes chilenos manifestaron su preocupación por la proyección de Chile a su mar adyacente. Inicialmente la dinámica se desarrolló a partir de motivaciones comerciales, impulsadas por privados que demandaron al Estado la apertura de consulados y oficinas de comercio (Echeverría, Orrego \& O'Shea, 1982), entre las cuales se puede contar Auckland, Melbourne, Sídney, Cantón, Hong Kong, Manila, Papeete, Honolulu, Bombay, Calcuta y Brunei, por citar algunos. También en el epistolario de Portales aparece prístina la vocación de preponderancia naval, que tanto lo distanciaría de los asuntos americanos (Sánchez, 1977).

La apertura de nuevos mercados en el Pacífico significó el inicio del tráfico comercial. Dicho tráfico tuvo, sin embargo, ciclos irregulares, altamente dependientes de variables domésticas y de la ecuación complementariedad-competitividad. Por ejemplo, en 1849 se descubrió oro en Australia, lo que trajo para Chile un aumento explosivo de la demanda de grano y harina (Barros, 1990). Esta tendencia sólo duró un lustro -hasta 1854 aproximadamente-, invirtiéndose a favor de los productores australianos que no sólo pudieron cubrir sus necesidades internas, sino que exportaron su trigo incluso a Chile.

Aunque el bombardeo español sobre Valparaíso y la destrucción de la flota mercante en 1866 asestó un duro golpe en este camino, la Guerra contra Perú y Bolivia entre 1879 y 1883 consolidó la supremacía chilena sobre el Pacífico Sur. La mayor expresión fue la navegación 
hacia la Polinesia, que culminó con la incorporación de Rapa Nui o Isla de Pascua entre 1888 y 1892, mediante la adquisición de títulos de propiedad a los jefes indígenas.

Un siglo más tarde, concretamente hacia 1994, Chile pasaría a formar parte de uno de los principales foros de la macro-región del Pacífico, la APEC (Asia Pacific Economic Cooperation) el cual se estableció en Camberra en 1989. Este foro reúne a algunas de las economías más dinámicas del orbe, bajo un programa flexible de fortalecimiento del sistema de comercio multilateral, bajo tres pilares fundamentales: las acciones voluntarias y unilaterales, la reciprocidad y el regionalismo abierto no discriminatorio.

Sin perjuicio de lo anterior, el antecedente inmediato de la Alianza del Pacífico se encuentra en la iniciativa Arco del Pacífico, lanzada el año 2007, con el objeto de congregar y hacer converger el comercio de once países ribereños del océano Pacífico en su promoción del intercambio con Asia. La Alianza recoge este objetivo y lo profundiza, buscando promover la libre circulación de bienes, servicios, capitales y personas entre sus países miembros (Chile, Perú, Colombia y México) y entre éstos y los mercados asiáticos. También cuenta como antecedente al Mercado Integrado Latinoamericano (MILA), que une las bolsas de valores de Chile, Colombia y Perú.

De esta situación emerge la Alianza del Pacífico, como un bloque inter-subregional, fundamentalmente comercial, sin la presencia de Estados Unidos, y como una sociedad entre Estados de similar nivel de desarrollo. Dicho conjunto tiene una adhesión manifiesta a una determinada visión de modelo económico, coherente con el tipo de acuerdos bilaterales de libre comercio que sus miembros han firmado con otros Estados. Observemos los acuerdos vigentes y suscritos por cada uno de los miembros originales de la Alianza:

Tabla 1. Acuerdos suscritos por los países miembros de la Alianza del Pacífico

\begin{tabular}{|l|l|l|}
\hline \multicolumn{1}{|c|}{ País } & \multicolumn{1}{c|}{ Vigentes } & \multicolumn{1}{c|}{ Suscritos } \\
\hline Chile & $\begin{array}{l}\text { Malasia, Turquía, Ecuador, Colombia, Australia, Perú, } \\
\text { Cuba, Panamá, Japón, India, P-4, EFTA, Corea, Estados } \\
\text { Unidos, Unión Europea, Centro América, México, } \\
\text { Canadá, Mercosur, Venezuela, Bolivia }\end{array}$ & $\begin{array}{l}\text { Hong Kong, Vietnam, } \\
\text { Tailandia }\end{array}$ \\
\hline Colombia & $\begin{array}{l}\text { CAN, CAN-MERCOSUR, Chile, México, Triángulo Norte } \\
\text { (Honduras, Guatemala y El Salvador), EFTA, Canadá, } \\
\text { CARICOM }\end{array}$ & $\begin{array}{l}\text { Unión Europea, Costa } \\
\text { Rica, Corea del Sur }\end{array}$ \\
\hline México & $\begin{array}{l}\text { Estados Unidos, Canadá, Costa Rica, Colombia, Nicaragua, } \\
\text { Chile, Israel, Guatemala, Honduras, El Salvador, Islandia, } \\
\text { Liechtenstein, Noruega, Suiza, Uruguay, Japón, Perú }\end{array}$ & \\
\hline CAN, MERCOSUR, APEC & $\begin{array}{l}\text { Venezuela, Costa Rica, } \\
\text { Guatemala }\end{array}$ \\
\hline
\end{tabular}

Fuente: Elaboración propia sobre la base de datos de las Cancillerías disponibles en la WEB. 
Sin embargo, dicha visión común no explicaría necesariamente la existencia de una disputa con otros bloques. La Alianza del Pacífico, creada en abril de 2011, surge de la iniciativa del ex Presidente Peruano Alan García de conformar un área de "integración profunda" entre los países miembros, entendida como la libre circulación de bienes, servicios, personas y capitales. En suma, posee una concepción fuertemente economicista de la integración, a diferencia de otros procesos nuevos que se desarrollan en la región, como es el caso de la UNASUR y el ALBA, caracterizados más bien por su signo fuertemente político y social.

Aunque la Alianza del Pacífico implica la reafirmación de la estrategia de regionalismo abierto como elemento fundamental de desarrollo, se hace cargo también de los cambios en la producción y el comercio mundial, por lo que no desdeña doctrinariamente las regulaciones económicas sobre el nivel doméstico, razón por la que Baldwin (2011) se refiere a este tipo de bloques y otros, como es el caso del Acuerdo de Asociación Transpacífica (TTP), como "Regionalismo del Siglo XXI". Además, la Alianza del Pacífico sería un ejemplo de la influencia que está teniendo la variable asiática en el comercio, las finanzas y la integración latinoamericana, en consonancia con las transformaciones globales que vive la geopolítica y la economía política mundial.

Según sostiene Mariano Turzi, en el fondo la Alianza pretende posicionarse frente a socios e inversores de Asia, a partir de las ventajas que ofrecerían sus cuatro miembros respecto de la facilitación de los intercambios y la integración financiera. Como señala este autor, de acuerdo con el informe Doing Business 2014 del Banco Mundial -que mide la facilidad de hacer negocios en el mundo-, los países de la Alianza del Pacífico están en los puestos número 34 (Chile), 42 (Perú), 43 (Colombia) y 53 (México). En contraste, los países del Mercosur ocupan puestos notablemente más bajos, con Uruguay en la ubicación 88, Brasil en la 116, Argentina en la 126, Paraguay en la 109 y Venezuela en la 181 (Turzi, 2014). A primera vista, las proyecciones de una y otra iniciativa se observan bastante diferentes.

Desde sus orígenes, la Alianza del Pacífico evidenció las políticas comerciales comunes de sus miembros de acuerdo a las normas del libre mercado, la desregulación económica y comercial, así como una pragmática noción del regionalismo abierto. A pesar de su corta existencia, la iniciativa ha generado grandes expectativas, especialmente ante la situación de que el PIB combinado de sus miembros representa aproximadamente el 35\% del total de América Latina y el Caribe, el octavo lugar en el comercio mundial.

Un tema relevante a señalar es que si bien la Alianza no cuenta con un liderazgo indiscutido, de todos modos es claro el peso determinante que ejerce México, al igual que el lugar predominante de Brasil en el MERCOSUR. Para el país norteamericano, la Alianza representa una oportunidad de acercarse hacia América Latina y los mercados de Asia ante la recurrente crítica que se le hace respecto de su virtual dependencia respecto de Estados Unidos.

La Alianza del Pacífico contó con el entusiasta apoyo de Chile desde sus inicios, atendida la cercanía ideológica de los supuestos de la iniciativa con los del gobierno de derecha de 
Sebastián Piñera. Esta administración ejerció la Secretaría Pro Témpore de la Alianza durante el año 2012, transformándose en uno de los hitos principales de Chile en materia de política exterior en dicho período. Los éxitos que ha exhibido la Alianza del Pacífico en el terreno comercial, llevaron al nuevo gobierno de Michelle Bachelet a continuar con el apoyo a este mecanismo, pero intentando conciliar dicha relación con su participación en el MERCOSUR, para atenuar las molestias de Brasil frente a la iniciativa del Pacífico y evitar impugnaciones ideológicas frente a la misma, entre otros objetivos.

\section{EL MERCOSUR}

El MERCOSUR tiene entre sus antecedentes una serie de encuentros y acercamientos entre los presidentes Raúl Alfonsín (Argentina) y José Sarney (Brasil), que lideraban los primeros procesos de transición democrática que se vivían en el Cono Sur en los ochenta. En 1986, los gobiernos de Buenos Aires y Brasilia firmaron un acuerdo de complementación industrial, basado en un mecanismo de integración económica. Asimismo, en los albores de la década del noventa, con el telón de fondo que entregaba el fin de la Guerra Fría y el Consenso de Washington, las administraciones de estos países optaron por un proceso de apertura económica hacia la economía global y de atracción de inversiones extranjeras a un mercado regional ampliado (Gardini, 2010). Los acercamientos finalmente se plasmaron en marzo de 1991, cuando los presidentes de estos países, junto a los de Paraguay y Uruguay, firmaron en Asunción el tratado del mismo nombre, mediante el cual se constituyó el MERCOSUR.

De este modo, el bloque se transformó en uno de los facilitadores de la tendencia regional, bautizada por Tokatlian y Rusell (2002), como la "autonomía relacional", orientada a promover la participación de los Estados de la región en los asuntos globales, mediante la participación en organizaciones y regímenes internacionales, de los cuales el MERCOSUR aparece como la viga maestra. Enseguida, como otros procesos de integración de la Posguerra Fría, con un correlato de vigorización de los espacios subnacionales (Maira, 2006), dicha instancia abrió las fronteras, colocando en estrecho contacto a gobiernos locales fronterizos de los Estados partes (Oddone, 2013). Así por ejemplo se potenciaron los Comités Ad Hoc que integran al día de hoy a sociedades civiles y políticas del centro y las periferias nacionales (Silva \& Morán, 2010), mediante las denominadas prácticas paradiplomáticas (Soldatos, 1990; Cornago, 2001).

En sus primeros años el bloque exhibió notables resultados, siguiendo los postulados del principio del regionalismo abierto. Según sostiene Natividad Lorenzo, desde su creación en 1991 y hasta 1998 el MERCOSUR evidenció importantes logros en la esfera comercial. En esta fase se logró liberalizar la totalidad de los productos comprometidos para esta etapa y aplicar un arancel externo común del 88\% del universo arancelario de la subregión. Todos estos avances atrajeron capitales y aumentaron la inversión extranjera directa. Sin embargo, la crisis económica y financiera que sufrió la región hacia fines de la década de los noventa, así como las dificultades para manejar las asimetrías existentes entre los Estados miembros o 
los problemas sociales endémicos, marcaron el fin de esta etapa favorable, iniciándose un nuevo periodo donde priman los intereses nacionales en las relaciones al interior del bloque, estancándose los flujos del comercio intrarregional. A ello se une la pérdida de confianza de los inversores extranjeros y la fuga de capitales (Lorenzo, 2006; Gardini, 2010; Gonçalves, 2013).

Desde entonces, el MERCOSUR se encuentra atado a un largo período de crítica y autorreflexión. Las quejas provienen principalmente de los socios menores del bloque, que cuestionan los reales beneficios de su pertenencia al proceso de integración, aduciendo que este no estaría respondiendo a sus intereses. Las dificultades del MERCOSUR, haciendo un esfuerzo de síntesis, se relacionan con la necesidad de reducir las asimetrías y disparidades entre los miembros, como el proteccionismo, así como con la vulnerabilidad externa de algunos de ellos. Los desafíos para el futuro, que no son fáciles de enfrentar y su solución aparece lejana, se refieren a la consecución de la unión aduanera (la literatura habla de la existencia de una indefinible unión aduanera imperfecta), el relanzamiento de las negociaciones comerciales y el eventual avance institucional.

Parte de los problemas del MERCOSUR derivan de las dificultades de conformar un claro liderazgo en la región. El eventual liderazgo brasileño no ha estado exento de problemas, por cuanto la plataforma regional de su posicionamiento global no está asegurada (Soares de Lima \& Hirst, 2006; Lechini \& Giaccaglia, 2007). Por lo demás, la entrada de Venezuela al bloque tampoco está clara desde la perspectiva de los liderazgos, en tanto su proyección se ha visto fuertemente debilitada a partir de la muerte de Hugo Chávez.

Asimismo cabe señalar que, si en un primer momento el MERCOSUR fue el símbolo del regionalismo abierto promovido por la CEPAL y en consecuencia coherente con los procesos de liberalización económica y desregulación que se vivían en el Cono Sur, la llegada de gobiernos de izquierda a los países ha planteado cambios en los modelos de desarrollo, los que se han proyectado al bloque, que actualmente está marcado por el proteccionismo y una fuerte crítica hacia la ortodoxia neoliberal. Sobre la base de un discurso regionalista sudamericano, Briceño observa que el bloque MERCOSUR ha privilegiado el crecimiento del mercado doméstico -lo que significa básicamente reindustrialización- unido a la promoción de los derechos sociales y laborales (Briceño, 2011). Este cambio en los lineamientos, así como la entrada de Venezuela, han provocado la mirada crítica desde Washington.

Ante el proceso de liberalización económica, comenzado por Chile desde el régimen militar y continuado por los gobiernos democráticos desde 1990, el país sólo entró al MERCOSUR en calidad de socio en 1996, en tanto su membresía plena era incompatible por las diferencias en las estructuras arancelarias y dañaría la anhelada autonomía comercial de Chile, por cuanto esto habría involucrado negociar conjuntamente con los demás miembros plenos ante terceros países. Sin perjuicio de estas trabas comerciales, el país ha participado activamente en los ámbitos políticos y sociales del bloque. 


\title{
LA CONVERGENCIA ENTRE LA ALIANZA DEL PACÍFICO Y EL MERCOSUR ¿POSIBILIDAD O NECESIDAD?
}

Como se ha señalado en este artículo, en materia de integración regional, uno de los principales postulados del segundo gobierno de Michelle Bachelet es la idea de la convergencia en la diversidad, particularmente entre la Alianza del Pacífico y el MERCOSUR. El Canciller Muñoz ha señalado sobre el particular que:

\begin{abstract}
Chile valora la Alianza del Pacífico como esquema de integración económica y plataforma comercial de proyección colectiva a la región de Asia Pacífico; pero no compartirá pretensión alguna de concebir dicha Alianza como un bloque ideológico excluyente o antagónico con otros proyectos de integración...Convergencia en la diversidad es la política que Chile buscará promover en América Latina. Es una opción que combina realismo y voluntad política de avanzar hacia una región más integrada y autónoma. (Muñoz, 2014)
\end{abstract}

En este orden de ideas, el Ministro Muñoz plantea que la región debiese discutir sobre la posibilidad de materializar la convergencia entre los mencionados bloques, considerando el concepto de la Unión Europea de las "velocidades diferenciadas". Ello permitiría que los países que estén en condiciones, y así lo deseen, puedan avanzar más rápido que los demás en el proceso de integración (Muñoz, 2014).

Si en un primer momento la Alianza del Pacífico generó críticas en Chile desde la oposición política al gobierno de Piñera, fuerte impulsor de esta iniciativa (la entonces oposición corresponde al actual gobierno, encabezado por Michelle Bachelet), y también cautelas por parte de otros países de la región, como es el caso de Brasil, que ven a la Alianza como opuesta al MERCOSUR y a la UNASUR, lo cierto es que el nuevo gobierno inaugurado en marzo de 2014, ante los positivos resultados de la instancia en materia comercial, actualmente la percibe como una oportunidad para apuntalar la presencia regional de Chile y su posicionamiento como puente hacia los mercados de Asia.

Probablemente, la aproximación entre la Alianza del Pacífico y el MERCOSUR comenzó a gestarse antes de la llegada al poder de Michelle Bachelet, prefigurada por una relación argentino-chilena que a pesar de ciclos de stop and go (Lorenzini, 2013), y de divergencias en las estrategias de inserción internacional y los modelos de desarrollo, supo preservar un espacio de convergencia bilateral (Aranda \& Ovando, 2014). En la aproximación entre los bloques, Buenos Aires jugó un papel crucial destacando la generación de un clima político favorable al diálogo de distintas iniciativas regionales, propiciado por la Presidenta argentina Cristina Fernández, durante la cumbre del MERCOSUR de julio de 2013. En el evento, Fernández abogó por el fortalecimiento de la concertación regional con respeto a los diversos modelos e instrumentos económicos que cada Estado asume en su camino al desarrollo (Busso, 2014). Esta sencilla declaración bien podría constituir un antecedente en el nuevo momento de las relaciones subregionales, en el 
sentido de una atenuación ideológica, donde los rasgos defensivos que adoptaron los bloques pudieran estar cediendo paso a una aproximación más funcional a los intereses conjuntos.

Por su parte, la estrategia chilena frente a la convergencia ha consistido en asumir a la Alianza de una manera pragmática, a fin de hacerla coherente con los procesos impulsados por los países de la costa atlántica de América del Sur. Este intento va de la mano de un fuerte acercamiento hacia Brasil, intento frustrado de la anterior presidencia de Sebastián Piñera. Para Chile, el coloso sudamericano representa un aliado estratégico en la región, cuyo liderazgo resulta esencial para apuntalar la integración. Esta idea fue claramente expresada por el Subsecretario de Relaciones Exteriores de Chile, Edgardo Riveros, durante el Coloquio Chile-Brasil, celebrado el 26 de agosto de 2014 en Sao Paulo. En la apertura del evento, Riveros planteó que "Brasil tiene que asumir un rol de liderazgo. No en vano es la gran potencia latinoamericana. No en vano participa de los BRICS y el G-20. Por eso esperamos que desempeñe un liderazgo gravitante en la región" (Ministerio de Relaciones Exteriores, 2014a). Desde una perspectiva más moderada, el asesor para asuntos internacionales de la presidencia brasileña, Marco Aurelio García, señaló que prefería hablar del liderazgo hegemónico colectivo, siguiendo la conceptualización planteada originalmente por el Barón de Río Branco, padre intelectual de la diplomacia brasileña, para referirse al pacto del ABC (Argentina, Brasil y Chile) de 1915 (W Radio, 2014, 26 de agosto). A este respecto, no se puede descartar que la Alianza del Pacífico llega en un momento en que Brasil se compromete gradualmente con Estados extra-regionales e intereses globales, perdiendo el MERCOSUR influencia relativa en la política exterior de Brasil, en favor de un regionalismo multilateral pragmático -a la manera de la UNASUR-o, si se prefiere, fortaleciendo el regionalismo para favorecer un sistema multipolar, según la teorización de Samir Amin (2005).

Diversos autores han vislumbrado como opuestos a la Alianza del Pacífico y el MERCOSUR, ante las evidentes diferencias de contenido y objetivos entre ellas. La Alianza del Pacífico se aprecia como pragmática en lo político, abierta en lo comercial y funcional a los intereses de Estados Unidos, desde el punto de vista de la geopolítica. Por su parte, el MERCOSUR aparece estancado -desde el punto de vista comercial, por cuanto el denominado "MERCOSUR político" sigue avanzando-, con un sesgo ideológico, fuertemente proteccionista y contestatario respecto de Washington (Turzi, 2014). En el mismo orden de ideas, Ignacio Bartesaghi señala que:

mientras Chile, Colombia, Perú y México, tienen acuerdos de libre comercio o de asociación con Estados Unidos, la Unión Europea (UE) y con otros países de Asia Pacífico (región a la que en los últimos años han definido como estratégica), los países del MERCOSUR priorizaron la integración más en clave política que económica (Unión de Naciones Suramericanas) y hasta la fecha no han cerrado acuerdos comerciales de impacto económico y comercial con Estados Unidos, Europa o Asia Pacífico. (Bartesaghi, 2014, p.44)

Sin perjuicio de lo anterior, se pueden destacar ciertas coincidencias. Por ejemplo, en su origen ambas instancias nacieron con elementos liberales de aperturismo económico, bajo un 
modelo de tipo regionalismo estratégico. A partir del nuevo siglo, el MERCOSUR agregó otros elementos sociales y de equidad, en consonancia con la rearticulación del modelo liberal democrático, en el cual el componente liberal se subordina a la idea de soberanía popular de la democracia (Busso, 2014), bajo un regionalismo híbrido que combina aspectos estratégicos, productivos y sociales (Briceño, 2013). Lo anterior ha acarreado un cierto desplazamiento de eje en el MERCOSUR, hacia posturas revisionistas del modelo de inserción liberal (Briceño, 2013), mientras la Alianza del Pacífico se mantiene bajo un predominio del principio del regionalismo abierto en el terreno comercial, agregando otros temas como el consular, científico y medio ambiental.

A pesar de que el MERCOSUR continúa siendo una unión aduanera imperfecta, se ha apegado al paradigma clásico de integración funcionalista que contempla, entre otros elementos, la constitución de bloques de países contiguos, mientras que la Alianza del Pacífico presenta una contigüidad geográfica relativa. Asimismo, el MERCOSUR es un proyecto cuyo núcleo gravitante es Brasil, con Argentina en un papel relevante, en un proyecto sudamericano; mientras la Alianza del Pacífico tiene un horizonte latinoamericano con participación de miembros de la CAN, el Sistema de Integración Centroamericano (SICA) e incluso el NAFTA, con México en un papel prominente.

Para contextualizar el análisis, es necesario señalar que en ambas vertientes de la región -pacífica y atlántica-, los países han expresado diversas estrategias de inserción internacional, como proyección de sus distintos modelos de desarrollo. Estas diferencias se ven acentuadas por las disputas que se aprecian entre México y Brasil por alcanzar una situación de liderazgo regional. Esta disputa, junto con la decisión brasileña de promover marcos más flexibles de asociación regional, ha llevado a Brasilia a impulsar la UNASUR, como una manera de favorecer una idea sudamericana de integración que distancie a los mencionados actores norteamericanos, particularmente a Washington, que por medio de su influencia en Colombia sigue presente en el escenario sudamericano. De esta manera, las diferencias entre la Alianza del Pacífico y el MERCOSUR formarían parte de ciertas vicisitudes por el liderazgo latinoamericano. Si bien ambos mecanismos no cuentan con un liderazgo definido e incontestado, lo cierto es que por sus dimensiones, el peso de México y Brasil respectivamente, resultan tanto incuestionables como relativamente equivalentes entre sí.

Una notable diferencia entre los dos bloques se aprecia en el terreno de la densidad institucional. El MERCOSUR, con todos sus problemas de funcionamiento, posee un desarrollo institucional con cierta madurez, aunque ello puede explicarse por los años que han pasado desde su creación. En la misma línea, por ejemplo, cabe recordar que como mecanismo de integración, el MERCOSUR se planteó la creación de una unión aduanera (aunque no ha logrado perfeccionarla), cuestión que no ha estado entre los objetivos de la Alianza del Pacífico, instancia mucho más liviana y pragmática desde el punto de vista institucional. Ello se relaciona con las diversas perspectivas de ambas instancias respecto del comercio internacional. 
La Alianza del Pacífico cuenta con una economía más abierta, lo que se evidencia al calcular el indicador de apertura comercial y los aranceles promedio de ambos procesos de integración. En cuanto a lo primero, la apertura comercial de la Alianza duplica a la del MERCOSUR, mientras que el arancel promedio NMF ponderado del MERCOSUR de 12,1\% es el doble al de la Alianza, con un 6,6\% (Bartesaghi, 2014).

Como complemento de lo anterior, Ignacio Bartesaghi ha realizado el siguiente cuadro, utilizando los indicadores de Doing Business y World Economic Forum, con el objeto de evidenciar las diferencias en la competitividad de los países de una y otra iniciativa:

Tabla 2. Competitividad Países Alianza del Pacífico y MERCOSUR

\begin{tabular}{|l|c|c|c|l|}
\hline \multicolumn{1}{|c|}{ País } & $\begin{array}{c}\text { Posición en } \\
\text { el Doing } \\
\text { Business } \\
\mathbf{2 0 1 3}\end{array}$ & $\begin{array}{c}\text { Índice de } \\
\text { competitividad } \\
\text { del World } \\
\text { Economic } \\
\text { Forum, 2013 }\end{array}$ & $\begin{array}{c}\text { Promedio de } \\
\text { los indicadores }\end{array}$ & Proceso de integración \\
\hline Chile & 37 & 34 & 36 & Alianza del Pacífico \\
\hline México & 48 & 55 & 52 & Alianza del Pacífico \\
\hline Perú & 43 & 61 & 52 & Alianza del Pacífico \\
\hline Colombia & 45 & 69 & 57 & Alianza del Pacífico \\
\hline Uruguay & 89 & 85 & 87 & Mercosur \\
\hline Brasil & 130 & 56 & 93 & Mercosur \\
\hline Paraguay & 103 & 119 & 111 & Mercosur \\
\hline Argentina & 124 & 104 & 114 & Mercosur \\
\hline Venezuela & 180 & 134 & 157 & Mercosur \\
\hline
\end{tabular}

Fuente: Bartesaghi (2014, p.50).

Como es posible observar, los mencionados indicadores de competitividad se inclinan notoriamente en favor de los países de la Alianza del Pacífico.

Por otro lado, ambos bloques tienen diferencias importantes en lo relativo a la estructura de las exportaciones. La Alianza aventaja al bloque del Cono Sur en 28 puntos porcentuales en la participación de las exportaciones con contenido tecnológico, aunque en gran medida ello se explica por la estructura exportable de México. Los demás países de la Alianza, en tanto, están marcados por la primarización de su comercio internacional (Bartesaghi, 2014).

Todo lo anteriormente señalado se puede complementar con la tipología planteada por Gardini (2010), que se enfoca en tres temas fundamentales de la integración regional, que se 
constituirían como importantes obstáculos de dicho proceso y, en este caso, particularmente para la convergencia entre la Alianza del Pacífico y el MERCOSUR, a saber: la cuestión del liderazgo, el posicionamiento ante Estados Unidos y el modelo de desarrollo. En tal sentido, es claro que la Alianza cuenta con México como actor predominante, es cercana a los intereses de Estados Unidos y el modelo de desarrollo que han seguido sus países, habida cuenta de los matices, podría calificarse como libremercadista. Por su parte, Brasil predomina marcadamente en el MERCOSUR, con un acento crítico hacia Estados Unidos y un modelo de desarrollo de carácter más endógeno y cercano al proteccionismo.

No obstante las señaladas diferencias -así como el hecho de que una y otra instancia responden a un liderazgo regional distinto (México en la Alianza y Brasil en el MERCOSUR)-, la oposición entre dichos procesos no parece funcional a los intereses de la región. La inserción global de América Latina requiere de un accionar conjunto y pragmático. Como señala Mariano Turzi, "la oposición entre MERCOSUR y AP (Alianza del Pacífico) es apresurada, intencionada y superficial. Modelos alternativos de integración no implican exclusiones y antinomias" (Turzi, 2014, p.82).

En este punto, la iniciativa chilena de impulsar la convergencia en la diversidad, con todas sus dificultades, parece muy adecuada para responder a las necesidades e intereses de la región en materia de desarrollo e inserción internacional. A fines de mayo de 2014, con ocasión de su participación en la XI Reunión de Cancilleres de la Alianza del Pacífico celebrada en México, el Ministro Muñoz, al momento de conceder una entrevista al periodista Andrés Oppenheimer, planteó en el mismo tenor: "no quisiéramos que la Alianza del Pacífico sea concebida como una suerte de bloque político excluyente, que se contraponga al Atlántico. Este no es el bloque de los librecambistas y eficientes, versus los estatistas ineficientes" (El Mercurio, 2014, 30 de mayo). La convergencia, a juicio del Ministro, podría explorarse, por ejemplo, en los ámbitos aduanero, turismo e integración cultural. En concordancia con lo anterior, en la declaración final de la reunión de la Alianza del Pacífico, a propuesta de Chile, se recogió la idea de ampliar el diálogo con los países del MERCOSUR.

Esta misma idea estuvo en el centro de la participación de la Presidenta Bachelet en la IX Cumbre de la Alianza del Pacífico, celebrada en junio de 2014 en Nayarit: "más allá de las legítimas diferencias, es perfectamente posible alcanzar niveles de convergencia entre los países de la Alianza y el MERCOSUR...No sólo es posible, es necesario" (El Mercurio, 2014, 20 de junio), señaló al inicio del evento. Como resultado de la propuesta chilena, el 1 de noviembre de ese año se celebró en Cartagena de Indias una reunión conjunta de ambos bloques, con miras a avanzar en la armonización de dichas instancias. Al respecto, dando muestras de moderación, el Canciller Muñoz señaló que "hay que ser gradual, pragmático y complementario. No se trata de estimular una fusión de los bloques. No está en la agenda" (El Mercurio, 2014, 2 de noviembre).

Avanzando en su iniciativa de convergencia, el 24 de noviembre de 2014 el gobierno chileno organizó en Santiago el seminario "Diálogo sobre integración regional: Alianza del Pacífico y MERCOSUR", que contó con la presencia de altas autoridades políticas y económicas de 
la región. Durante su intervención, el Canciller chileno señaló, entre otros aspectos, que el MERCOSUR y la Alianza del Pacífico responden a modelos económicos y formas distintas de inserción en la economía. No obstante ello, constituyen dos componentes esenciales cuya convergencia gradual y pragmática otorgaría importantes beneficios a los países integrantes de cada uno de estos bloques (Ministerio de Relaciones Exteriores, 2014b). Durante el evento de Santiago destacó la definición de una agenda corta para comenzar a trabajar en la convergencia de ambas iniciativas, partiendo por los sectores menos complejos y fáciles de trabajar, como en el ámbito del intercambio de bienes culturales, ventanillas únicas y movilidad de personas. En suma, se trataría de propiciar una integración gradual y pragmática, dejando de lado por el momento el tema arancelario. En el fondo, se trataría de avanzar en la coherencia e integración de ambos bloques, antes que de una fusión de los mismos.

Desde la perspectiva de este artículo, pese a los avatares que se aprecian en este proceso de convergencia, éste debe asumirse como una necesidad para los intereses de los países y para la región en su conjunto. Más que preguntarse si es posible la convergencia en la integración regional, debe reflexionarse sobre este proceso como una necesidad. Pese a las diferencias existentes, el mundo actual exige pragmatismo en las conductas de los variados actores internacionales y transnacionales. Los países, y en ellos especialmente quienes están a cargo de la toma de decisiones en materia de política exterior, deben concebir una región plural y diversa, con un marco regional multilateral en el que se puedan expresar diferencias y afinidades en variadas temáticas, en virtud de los diversos foros existentes. Es que, pese a las divergencias que se aprecian entre la Alianza del Pacífico y el MERCOSUR, ello no implica que los miembros de dichas instancias no puedan ponerse de acuerdo en ciertos temas con el objeto de proyectar a la región en el ámbito internacional.

La presencia y el virtual poder que posea América Latina a nivel global, depende de que actúe fragmentada o conjuntamente, en apoyo de las reglas propias del multilateralismo y en función de sus intereses como un actor fundamental de aquella sección del mundo denominada Sur.

\section{CONCLUSIÓN}

En una columna aparecida en The Wall Street Journal en enero de 2014, reproducida por El Mostrador (2014, 6 de enero), se insistía en la fractura en dos de la región de América Latina. Un bloque que colinda con el Atlántico, representado por Brasil, Argentina y Venezuela, desconfiaría de la globalización, otorgaría al Estado un papel de alta relevancia en la economía y sería hostil hacia Washington. Por otro lado, ciertos países del Pacífico, como México, Perú, Chile y Colombia, promoverían el libre comercio y la apertura de los mercados, manteniendo estrechos lazos con Estados Unidos.

Sin perjuicio de la validez de tales argumentos, esta división de la región parece poco útil para los intereses y objetivos de la región latinoamericana. Actualmente, el poder depende cada vez 
más de la capacidad de los países de actuar de manera coordinada y con una voz común en la economía global y la política multilateral. Una cita de Joseph Tulchin y Ralph Espach, resulta especialmente útil en esta línea:

Para aquellos países que gozan de escaso poder en sentido realista, como sucede con los latinoamericanos, la participación en las instituciones internacionales difunde el poder y proporciona a los países un escenario para la negociación en donde pueden acrecentar su influencia en lo relativo a la construcción de los valores y reglas institucionales. (2004, pp.28-29)

De lo anterior, se desprende que avanzar de manera pragmática hacia la convergencia entre ambos bloques es una oportunidad para superar la fragmentación de la región, más allá de definiciones o adhesiones en torno a los diversos regionalismos que han sido revisados en este artículo. Como señala el Canciller chileno Heraldo Muñoz, los ámbitos aduanero, turístico y cultural, pueden representar una oportunidad para este fin superior y una plataforma para comenzar a abordar otras áreas más sensibles.

Pese a las diferencias que evidencian la Alianza del Pacífico y el MERCOSUR, el carácter marcadamente económico de la primera y el asumido perfil político que ha adquirido el segundo, no constituyen un óbice insalvable para alcanzar una complementariedad que apuntale la inserción global de la región.

En la región, la dinámica de diálogo político que otorgan las diversas instancias de integración ha facilitado el procesamiento de las diferencias por medio del entendimiento presidencial y ministerial. Dicho derrotero podría permitir articular los consensos necesarios para enfrentar exitosamente los desafíos contemporáneos que supone la inserción regional en el contexto internacional. De tal modo, las discrepancias entre los países son procesadas por medio de mecanismos políticos, lo que sugiere un rol renovado para los actores estatales, más allá de las diferencias entre aquellos proclives a posturas nacionalistas o aquellos con una orientación internacionalista.

Chile, consciente de su peso específico, pero sobre todo de los beneficios de una activa política multilateral en diversos niveles y sin abandonar su estrategia de regionalismo abierto, ha enfatizado su papel de bisagra en dos proyectos regionales que, con énfasis y estrategias diversas, no prescinde del contexto internacional como variable a considerar en la facilitación del desarrollo doméstico. En tal contexto, el interés prioritario de este país es propiciar una integración regional inclusiva y amplia, que promueva el trabajo colectivo en torno a consensos y respetando las diferencias de cada país. Bajo este enfoque, se hace urgente superar los esquemas conformacionales rubricados bajo las imágenes de ejes y alianzas antagónicas, para pasar a atender a objetivos e intereses comunes en materia de inserción internacional. Para Chile, así como para la región en su conjunto, la convergencia en la integración no es una cuestión de posibilidades, sino una necesidad. 


\section{REFERENCIAS}

Actis, E. (2014). Brasil y sus pares sudamericanos de la Alianza del Pacífico. Divergencias regionales en las opciones nacionales e internacionales. Relaciones Internacionales, 46, 71-87.

Amin, S. (2005). Más allá del capitalismo senil. Por un siglo XXI no norteamericano, Buenos Aires: Paidós.

Aranda, G. \& Salinas, S. (2015). ALBA y Alianza del Pacífico: ¿Choque de integraciones? Universum. 30(1). en imprenta.

Aranda, G. \& Ovando, C. (2014). Estrategias de autonomía e integración convergiendo en una relación bilateral. El caso de la Argentina y de Chile. En Briceño, J. \& Simonoff, A. (Eds.) Integración y cooperación regional en América Latina. Una relectura a partir de la teoría de la autonomía (pp. 243-261). Buenos Aires: Editorial Biblos.

Aranda, G. \& Riquelme, J. (2011). La política exterior de Chile desde 1990. Inserción internacional y prioridad regional. Cuadernos sobre Relaciones Internacionales, Regionalismo y Desarrollo, 6(11), 11-41.

Bachelet, M. (2013). Programa de Gobierno Michelle Bachelet 2014-2018. Recuperado de http://www.minsegpres.gob.cl/wp-content/upLoads/2014/04/ProgramaMB.pdf

Baldwin, R. (2011). 21st Century Regionalism: Filling de Gap between 21st century trade and 20st century trade rules. World Trade Organization. Recuperado de http://www.wto.org/ english/res_e/reser_e/ersd201108_e.pdf

Barros, M. (1990). Historia Diplomática de Chile. Santiago de Chile: Editorial Andrés Bello.

Bartesaghi, I. (2014). El MERCOSUR y la Alianza del Pacífico, ¿más diferencias que coincidencias? Mundo Asia Pacífico, 3(1), 43-56. Recuperado de http://publicaciones.eafit.edu.co/index. $\mathrm{php} / \mathrm{map} /$ article/view/2481/2370

Bernal-Meza, R. (2011). Heterodox Autonomy Doctrine: realism and purpose, and its relevance. Revista Brasileira de Política Internacional, 2(56), 45-62.

(2013). América Latina en el mundo. El pensamiento latinoamericano y la teoría de las relaciones internacionales. Buenos Aires: Grupo Editor Latinoamericano.

Bizzozero, L. (2011). Aportes del MERCOSUR al regionalismo y a la teoría política de la integración regional. Una mirada desde los veinte años del proceso. Boletín Meridiano 47, 12(125), 4-11. 
Briceño, J. (2011). Del regionalismo estratégico al regionalismo social y productivo. Las transformaciones del modelo de integración en el MERCOSUR. En Briceño, J. (Ed.), El Mercosur y las complejidades de la integración regional (pp. 121-162). Buenos Aires: Teseo.

Briceño, J. (2013). Ejes y modelos en la etapa actual de la integración económica regional de América Latina, Estudios Internacionales, (175), 9-39.

Bouzas, R. (1997). Regionalización e Integración Económica, Instituciones y Procesos Comparados. Buenos Aires: Grupo Editor Latinoamericano.

Busso, A. (2014). Los vaivenes de la Política Exterior Argentina re-democratizada (1983-2013). Los impactos de los condicionantes internos. Estudios Internacionales, (177), 9-33.

Cornago, N. (2001). Diplomacia, paradiplomacia y redefinición de la seguridad mundial: dimensiones de conflicto y cooperación, en Aldecoa, F. \& Keating, M. (Eds.). Paradiplomacia: las relaciones internacionales de las regiones (pp. 55-77). Madrid: Marcial Pons.

Echeverría, G., Orrego F. \& O’Shea, L. (1982). Los intereses de Chile en el Pacífico. Revista de Educación, (94).

El Mostrador (2014, 6 de enero). La historia económica de dos Latinoaméricas. Recuperado de http:// www.elmostradormercados.cl/destacados/la-historia-economica-de-dos-latinoamericas/

Gardini, G. (2010). Proyectos de integración regional sudamericana: hacia una teoría de convergencia regional. Relaciones Internacionales, (15), 11-31. Recuperado de http:// www.relacionesinternacionales.info/ojs/article/view/237.html

Gonçalves, J. (2013). Aliança estratégica entre Brasil e Argentina: antecedentes, estado atual e perspectivas. En Paz, L. (Org.) O CEBRI e as relações internacionais no Brasil (pp. 41-67). São Paulo: SENAC.

Hettne B. \& Söderbaum F. (2002). Theorizing the rise of regions. En Breslin, S. et. al. (Eds.), New Regionalim in the Global Political Economy. Theories and Cases (pp. 33-47). London: Routledge.

Hurrell, A. (1995). Explaining the resurgence of regionalism in World Politics. Review of International Studies, 21(4), 331-368.

Lechini, G. \& Giaccaglia, C. (2007). El Brasil en el laberinto de los espejos. Su rol regional en el siglo XXI, El Debate Político. Revista Iberoamericana de Análisis Político, (6-7), 70-87.

Lorenzini, M. (2013). Las relaciones argentino-chilenas 2008-2011 ¿Realidad o ficción de la Alianza Estratégica?, Si Somos Americanos. Revista de Estudios Transfronterizos, 13(1), 39-64. 
Lorenzo, N. (2006). Situación actual y perspectivas del MERCOSUR, Madrid: Fundación CILAE. Recuperado de http://www.cilae.org/publicaciones/NT0306.pdf

Maira, L. (2006). Las relaciones entre Argentina y Chile: un caso singular de combinación entre los vínculos interestatales y la paradiplomacia. En Rodríguez, S. (ed.). La Paradiplomacia: las relaciones internacionales de los gobiernos locales (pp. 83-114), México: Cámara de Diputados.

Malamud, A. (2009). Divergencias en ascenso: viejas y nuevas fracturas en América Latina. Araucaria. Revista Iberoamericana de Filosofía, Política y Humanidades, (21), 125-139.

Ministerio de Relaciones Exteriores, Gobierno de Chile (2010). Perspectivas, proyecciones y desafíos de la política exterior de Chile. Santiago: Gobierno de Chile.

(2014a). Cancilleres y Ministros de la Alianza del Pacífico y MERCOSUR se juntaron en Santiago para dialogar sobre la integración regional. Recuperado de http://www.minrel.gov.cl/cancilleres-y-ministros-de-la-alianzapacifico-y-mercosur-se-juntaron-en-santiago-para-dialogar-sobre-la-integracion-regional/ minrel/2014-11-24/141939.html

Riveros subrayó necesidad de liderazgo brasileño en fortalecimiento de integración regional, en Coloquio Chile-Brasil en Sao Paulo. Recuperado de http://www.minrel. gob.cl/subsecretario-edgardo-riveros-subrayo-necesidad-de-liderazgo-brasilenoen-fortalecimiento-de-integracion-regional-en-coloquio-chile-brasil-en-sao-paulo/ minrel/2014-08-26/180202.html

Morandé, J. (1999). Globalización y regionalismo en el nuevo escenario internacional. Santiago de Chile: Mimeo.

Muñoz, H. (2014, 13 de marzo). Convergencia en la diversidad: la nueva política latinoamericana de Chile. Diario El País.

Nanjira, D. (2010). African Foreign Policy and Diplomacy (Vol. 2), Santa Bárbara: Praeger.

Nolte, D. (2007). Potencias regionales: conceptos y enfoques de análisis. El Debate Político. Revista Iberoamericana de Análisis Político, (6-7). 17-35.

Oddone, N. (2013). Una paradiplomacia transfronteriza para la geografía de la(s) América(s) Latina(s). En Zeraoui, Z. (Ed), Teoría y práctica de la paradiplomacia (pp. 119-147). Monterrey: ITESM. 
Perrotta, D. (2013) La integración regional como objeto de estudio. De las teorías tradicionales a los enfoques actuales. En Llanderrozas, L. (ed.), Relaciones Internacionales. Teorías y debates (pp. 197-252). Buenos Aires: EUDEBA.

Piñera, S. (2009). Programa de Gobierno para el cambio, el futuro y la esperanza. Chile 20102014. Recuperado de http://www.sebastianpinera.cl/sites/default/files/programa_de_ gobierno_2010.pdf

Portales, C. (2011). Desafíos para la política exterior en las próximas décadas: Chile y la región en un mundo global. Estudios Internacionales, (169), 171-193.

Rojas, F. (2013). Regionalismo e Integración Regional. En Legler, T., Santa Cruz, A. \& Zamudio, L. (Eds.), Introducción a las Relaciones Internacionales: América Latina y la Política Global (pp. 158-171). México: Oxford University Press.

Sanahuja, J. (2008). Del regionalismo abierto al regionalismo posliberal. Crisis y cambio en la integración en América Latina y el Caribe. En Martínez, L. et. al. (Eds.), Anuario de la integración regional de la América Latina y el Gran Caribe 2008-2009, Buenos Aires: CRIES.

Sánchez, W. (1977). Las tendencias sobresalientes de la Política Exterior chilena. En Sánchez, W. (ed.), 150 años de Política Exterior Chilena (pp. 374-421). Santiago de Chile: Editorial Universitaria.

Silva, V. \& Morán, C. (2010). Relaciones subnacionales. El caso de Chile-Argentina, en Maira, L. (Ed.). La política internacional subnacional en América Latina (pp. 259-303). Buenos Aires: Libros el Zorzal.

Soares de Lima, M. \& Hirst, M. (2006). Brazil as an Intermediate State and Regional Power: Action, Choice and Responsibilities. International Studies, 82(1), 21-40.

Soldatos, P. (1990). An explanaroty framework for the study of federal states as foreign-policy actors. En Michelmann H. y Soldatos, P. (Eds.), Federalism and international relations. The role of subnational units (pp. 34-53). Oxford: Clarenton Press.

Tokatlian, J. \& Rusell, R. (2002). De la autonomía periférica a la autonomía relacional: una mirada teórica desde el Cono Sur. Perfiles Latinoamericanos, (21).

Tulchin, J. \& Espach, R. (2004). América Latina en el nuevo sistema internacional: la necesidad del pensamiento estratégico. En Tulchin, J. \& Espach, R. (Eds.), América Latina en el nuevo sistema internacional (pp. 21-66). Barcelona: Bellaterra.

Turzi, M. (2014). Asia y la ¿(des) integración latinoamericana? Nueva Sociedad, (250), 78-87. 
Van Klaveren, A. (2014). El análisis de la política exterior: Una visión desde América Latina. En Legler, T., Santa Cruz, A. \& Zamudio, L. (Eds.), Introducción a las Relaciones Internacionales: América Latina y la Política Global, México (pp. 96-109). Oxford: University Press.

W Radio (2014, 26 de agosto). Chile se ofrece en Brasil como puente entre MERCOSUR y Alianza del Pacífico. Recuperado de http://www.wradio.com.co/noticias/internacional/ chile-se-ofrece-en-brasil-como-puente-entre-mercosur-y-alianza-del-pacifico/20140826/ nota/2384207.aspx

Wilhelmy, M. \& Durán, R. (2003). Los principales rasgos de la política exterior chilena entre 1973 y el 2000. Revista Ciencia Política, 23(2), 273-286. 